Check for updates

Cite this: Chem. Commun., 2020,

56, 1689

Received 6th December 2019,

Accepted 9th January 2020

DOI: $10.1039 / c 9 c c 09507 a$

rsc.li/chemcomm

\section{Control of polymorphism in thiophene derivatives by sublimation-aided nanostructuring $\dagger$}

\author{
Denis Gentili, (D) a Ilse Manet, (DD ${ }^{b}$ Fabiola Liscio, (D) ${ }^{c}$ Marianna Barbalinardo, (D) ${ }^{a}$ \\ Silvia Milita, ${ }^{c}$ Cristian Bettini, ${ }^{\mathrm{b}}$ Laura Favaretto, ${ }^{\mathrm{b}}$ Manuela Melucci, ${ }^{\mathrm{b}}$ \\ Alessandro Fraleoni-Morgera (D) ${ }^{d}$ and Massimiliano Cavallini (D) ${ }^{a}$
}

\begin{abstract}
Here we applied a novel concept of "sublimation-aided nanostructuring" to control the polymorphism of a model material. The process exploits fractional precipitation as a tool for crystallisation in confinement using a templating agent that sublimes away from the system at the end of the process.
\end{abstract}

Polymorphism, i.e. the existence of different forms or crystallised structures of a solid substance with the same stoichiometry, is a wellknown phenomenon relevant to all scientific disciplines dealing with solid materials. ${ }^{1,2}$ Although in some cases polymorphism brings added value as it originates new functional properties ${ }^{3}$ or tunes existing ones, ${ }^{4,5}$ usually, solid-state applications require the controlled selection of a specific polymorph. ${ }^{6}$

Several methods have been proposed for control of polymorphism, including chemical design, ${ }^{7}$ processing, ${ }^{8-10}$ templating via heterogeneous nucleation, ${ }^{11}$ crystallisation kinetics control, ${ }^{12}$ post-deposition treatments, ${ }^{13}$ crystallization in confinement ${ }^{14-16}$ and others. ${ }^{17-19}$ These methods are discussed in detail in many excellent articles and dedicated reviews.,15,17-19 Among the proposed approaches, deposition in confinement is particularly appealing, since it has been proved to be efficient in polymorph selection in a lot of cases; ${ }^{14,18-23}$ moreover, it is integrable with several conventional and unconventional fabrication techniques, which helps the polymorph's implementation in devices. ${ }^{24,25}$ Crystallisation in confinement is performed by three principal approaches; all of them use an external physical tool to confine the crystallisation process: inside a nano (meso)-porous solid matrix, ${ }^{18,19,22}$ by solution shearing, ${ }^{14,20,23}$ and by wet lithography. ${ }^{16,26}$

\footnotetext{
a Istituto per lo Studio dei Materiali Nanostrutturati (ISMN), CNR, Via Gobetti 101, 40129 Bologna, Italy. E-mail: massimiliano.cavallini@cnr.it

${ }^{b}$ Istituto per la Sintesi Organica e la Fotoreattività, CNR, Via Gobetti 101, 40129 Bologna, Italy

${ }^{c}$ Istituto per la Microelettronica e Microsistemi, CNR, Via Gobetti 101, 40129 Bologna, Italy

${ }^{d}$ Dipartimento di Ingegneria e Architettura, Università di Trieste, Via Valerio 10, 34100 Trieste, Italy

$\dagger$ Electronic supplementary information (ESI) available: Experimental and characterization details and additional figures. See DOI: 10.1039/c9cc09507a
}

Among the proposed approaches, the use of a nano(meso)porous solid matrix is the most studied confinement process for polymorph selection; it was performed in several types of matrix such as zeolites, ${ }^{22}$ polymers,${ }^{18}$ microgels, ${ }^{19}$ and porous glasses. ${ }^{18,19}$ The approach is very efficient and versatile, however the separation of the polymorphs from the matrix is an open problem, especially if the polymorph must be implemented in a device after its selection.

In polymorph selection by solution shearing a plate shears a thin film of a solution, placed on a substrate on which the crystallization occurs during the shearing process. ${ }^{14,20,23}$ Solution shearing is very efficient and allows the selection and positioning of specific polymorph thin films directly into devices. However, it is available for a limited number of materials and requires dedicated instrumentation.

In wet lithography, crystallization in confinement is obtained inside an artificial micro/nano channel fabricated by an appropriate polymeric stamp. ${ }^{6,24}$ Differently from solution shearing, wet lithography allows the fabrication of geometrically defined micro/nano structures. Wet lithography is versatile and very efficient allowing the selection and positioning of the polymorph directly into devices, but as in the case of solution sharing, it requires a specific instrumentation set-up.

Here we show a full-chemical approach based on the use of a matrix made of a sublimating compound, which is spontaneously eliminated from the system at the end of the process by sublimation. The proposed approach paves the way for a new, simple, route for the control of polymorphism by confinement, eliminating the problem of separating the polymorph from the confining matrix. We used "Auxiliary Solvent-Based Sublimation-Aided Nanostructuring (ASB-SANS)”, a recently developed method used to grow self-assembled nanostructures of a target material (1) by drop-casting $^{27-30}$ schematised in Fig. 1.

In ASB-SANS, a solution of the target material (1) and of a compound capable of sublimating under ambient conditions (sublimating compound, 2), overabundant in 2, is drop-cast on a substrate (Fig. 1a). When the solvent shrinks, the overabundant 2 reaches supersaturation and precipitates on the substrate, 

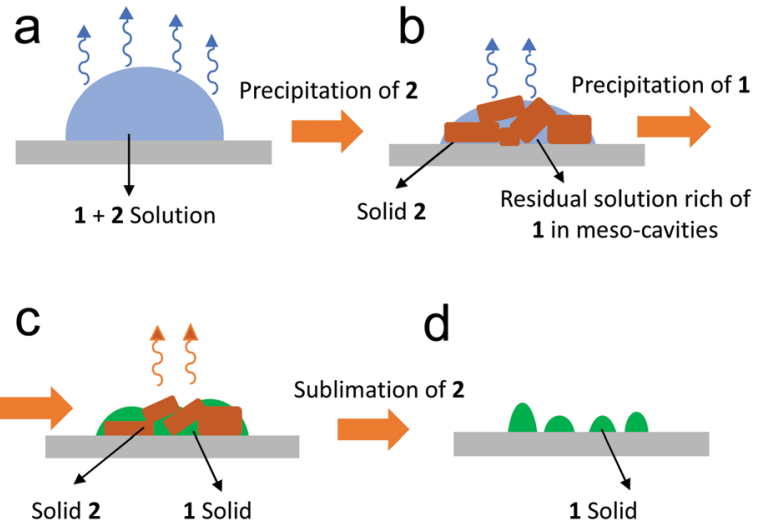

Fig. 1 Scheme of sublimation-aided nanostructuring (ASB-SANS). (a) Drop casting of $\mathbf{1}+\mathbf{2}$ solution, overabundant in $\mathbf{2}$ with a fast solution shrinking. (b) When overabundant 2 reaches supersaturation, it starts to precipitate forming a pattern of templating crystals, which decrease the solution shrinking and form the mesoscopic cavities. (c) When also compound 1 reach supersaturation it precipitates into the cavities, in confinement. (d) After that, $\mathbf{2}$ sublimates, leaving the nanostructured deposit of 1 on the substrate.

forming a 3D pattern of crystallites, which can incorporate some 1, with mesoscopic cavities in between them, filled with the residual solution rich in $\mathbf{1}$ (Fig. 1b). When also the solution of 1 reaches supersaturation, the pattern of $\mathbf{2}$ acts as a template for the precipitation of $\mathbf{1}$, which occurs inside the mesoscopic cavities (Fig. 1c) in between the 2 crystallites. Under ASB-SANS conditions (mesoscopic spatial confinement combined with slow solvent evaporation due to the presence of the 2 crystals), the solute precipitates under quasi-equilibrium and confinement conditions. After that $\mathbf{2}$ sublimates away, leaving only $\mathbf{1}$ structures on the surfaces (Fig. 1d). Here we exploited the combination of these capabilities to select a polymorph of a model compound. As a model compound (1) we used thieno(bis)imide end-functionalized terthiophene (Scheme 1), whose synthesis is described elsewhere. ${ }^{31}$

$\mathbf{1}$ is a well-characterized thermochromic material used in organic optoelectronics ${ }^{31}$ and in time-temperature integrator devices, ${ }^{26,32}$ capable of forming two types of polymorphs, discernible by the fluorescence colour. ${ }^{9,33}$ As sublimating compound 2 we used $p$-dichlorobenzene, a commercial substance that sublimes under ambient conditions, and whose effectiveness in ASB-SANS processes has already been demonstrated. ${ }^{27-30}$ The two substances were chosen for their ability to achieve separate precipitation from the solution, due to their different solubility under the specific experimental conditions.

Samples were prepared by drop-casting $10 \mu \mathrm{l}$ of a $\mathbf{1}+2$ solution ( $1=1 \mathrm{~g} \mathrm{~L}^{-1}, 2=300 \mathrm{~g} \mathrm{~L}^{-1}$ in chloroform and toluene)

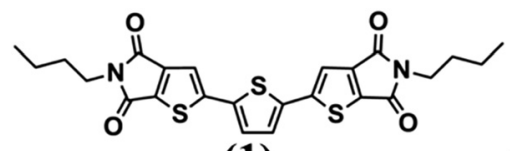

(1)

(2) ${ }^{\mathrm{Cl}}$

Scheme 1 Chemical structure of thieno(bis)imide end-functionalized terthiophene (1) and p-dichlorobenzene (2).

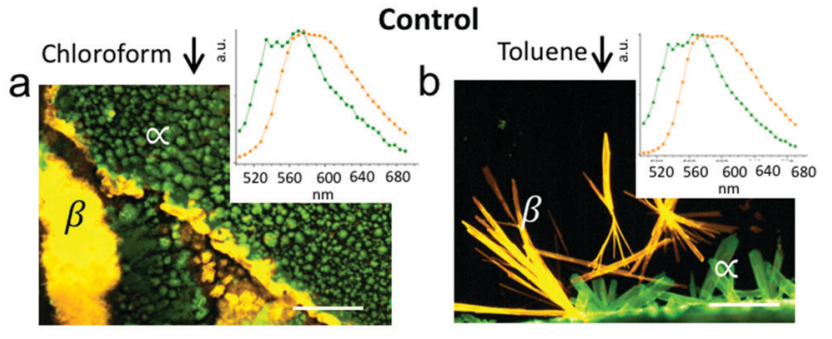

ASB-SANS

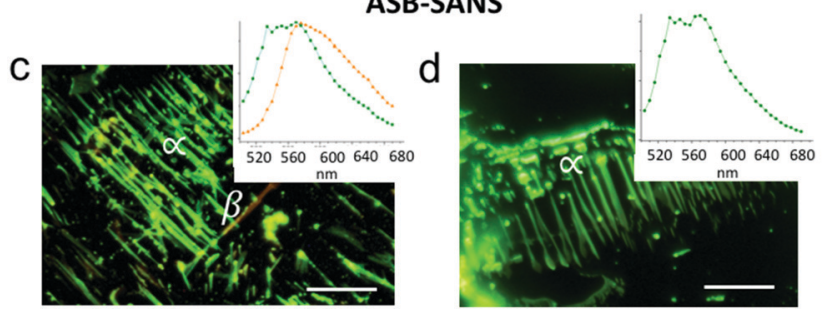

Fig. 2 Fluorescence microscopy images and corresponding fluorescence spectra of thin deposits of 1 on silicon. The insets show the fluorescence spectra recorded by confocal microscopy. In the insets the green curve refers to $\alpha$ polymorphs, and the orange curve refers to the $\beta$ polymorph. (a) Control sample deposited from chloroform and (b) control sample deposited from toluene. (c) ASB-SANS deposited from chloroform. (d) ASBSANS deposited from toluene. Scale bars are $100 \mu \mathrm{m}$.

on the substrate. Control samples were prepared by dropcasting $10 \mu \mathrm{l}$ of 1 solution $\left(1 \mathrm{~g} \mathrm{~L}^{-1}\right)$ in chloroform and toluene, respectively. All samples were deposited on $\mathrm{Si} / \mathrm{SiO}_{2}$ (thermal) in air and were characterized by fluorescence microscopy, X-ray diffraction, and confocal fluorescence microscopy.

When drop cast from a solution composed by only the solvent and itself (control samples), $\mathbf{1}$ forms two types of polymorphs: a polymorph (here named $\alpha$ ) that displays green fluorescence, thermodynamically stable at room temperature, ${ }^{9}$ and a polymorph (here named $\beta$ ) that displays yellow/orange fluorescence. ${ }^{9}$ Fig. $2 \mathrm{a}$ and $\mathrm{b}$ shows typical fluorescence microscopy images and confocal fluorescence spectra of the control samples, deposited from chloroform and toluene, respectively.

In the control samples, the percentage of polymorphs, size and crystal shape depend on the solvent. ${ }^{9}$ In particular, the polymorph percentage, measured from fluorescence images, ranges from $40 \%$ to $75 \%$ of $\alpha$ when deposited from chloroform (Fig. 2a) and from $55 \%$ to $80 \%$ of $\alpha$ when deposited from toluene (Fig. 2b). The size of the crystals ranges from a few tens of microns for the samples obtained from chloroform to a few hundred microns for the samples obtained from toluene. Remarkably, using ASB-SANS we obtained a dramatically preponderant formation of the $\alpha$ polymorph independently from the used solvent, namely $95 \pm 5 \%$ using chloroform (Fig. 2c) and $98 \pm 2 \%$ using toluene (Fig. 2d). Moreover, while in control samples the morphology of the deposited material differs significantly upon using toluene or chloroform solution (Fig. 2a and b), using ASB-SANS the morphology of the developed $\alpha$ phase appears to be the same independent from the used solvent (Fig. 2c and d). The comparison between the fluorescence spectra in the control samples and the samples prepared by ASB-SANS (insets of Fig. 2) shows that the samples 
are still composed of the same types of polymorphs, as the spectral shape is unaltered for all samples. Polymorph selection was not observed using a solution with a $1: 2$ ratio $<1: 100$ (i.e. without the initial precipitation of 2). Eventually, in order to exclude that the endothermic sublimation process induces the solid-state transformation we performed two control experiments.

(i) We cooled a control sample of $\mathbf{1}$ containing concomitant $\alpha$ and $\beta$ polymorphs to $-25{ }^{\circ} \mathrm{C}$. (ii) We prepared control samples at $-25{ }^{\circ} \mathrm{C}$.

In the first experiments, we did not observe any effect of cooling on polymorph composition. In the second experiment we observed a small increase of the $\alpha$ percentage (3-5\% more), which we attributed to the slower solvent evaporation. ${ }^{9}$

In order to gain more information of the morphology control exerted by the ASB-SANS procedure, we investigated samples of 1 by 2D Grazing Incidence X-ray diffraction (GIXRD) and high-resolution specular XRD (Fig. 3).

2D-GIXRD images show the presence Bragg peaks ascribed to the " $\alpha$ " polymorph structure, regardless of the solvent and the preparation method. In the case of control samples from chloroform solution (Fig. 3a), the small arc shape of the spots indicates a [100] texturing, while, when the sample is prepared by ASB-SANS, the 2D-GIXRD image also shows Debye rings (Fig. 3b), revealing the presence of randomly oriented crystal grains of the $\alpha$ polymorph.
In the case of toluene solution, the arc shape of the spots indicates a [100] texturing for both the control samples and ASB-SANS (Fig. 3d and e). However, the specular XRD pattern recorded for the samples prepared by ASB-SANS shows only the peaks of the $\alpha$ polymorph, whereas that for the thin deposits prepared by drop casting shows two additional Bragg peaks (Fig. 3f) indicating the presence of the $\beta$ polymorph.

We explained the polymorph selection exerted by ASB-SANS on the basis of two critical conditions that this process creates during the nucleation step of crystallisation of $\mathbf{1}$. The first one is the low evaporation rate of the solvent due to the presence of 2 acting as a capping layer (Fig. 1a) allowing the crystallization of 1 to occur under quasi-equilibrium conditions. This situation limits the molecular diffusion and increases the energy available for molecular motions during the crystallization, thus decreasing the number of nuclei of $\mathbf{1}$ and favouring the growth of the mean size of the crystalline domains. In addition, the confinement at the mesoscale of $\mathbf{1}$ exerted by the $\mathbf{2}$ crystallites can discriminate between different polymorphs on the basis of their different critical nuclei size. ${ }^{18,19,21}$ Dedicated investigations about the mechanism of polymorph selection are on-going. Regarding the morphology, as ASB-SANS goes through a first precipitation of 2, and a subsequent precipitation of $\mathbf{1}$, the morphology of $\mathbf{1}$ is templated by the $\mathbf{2}$ crystals, which are elongated needles organized in randomly oriented domains independently from

\section{Chloroform}
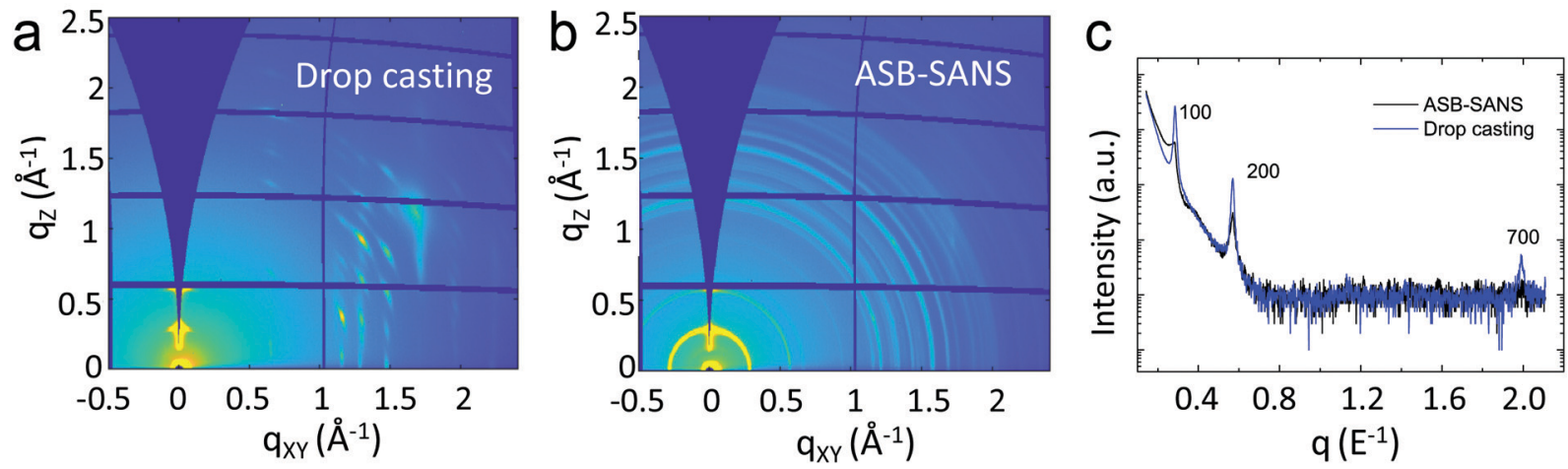

Toluene
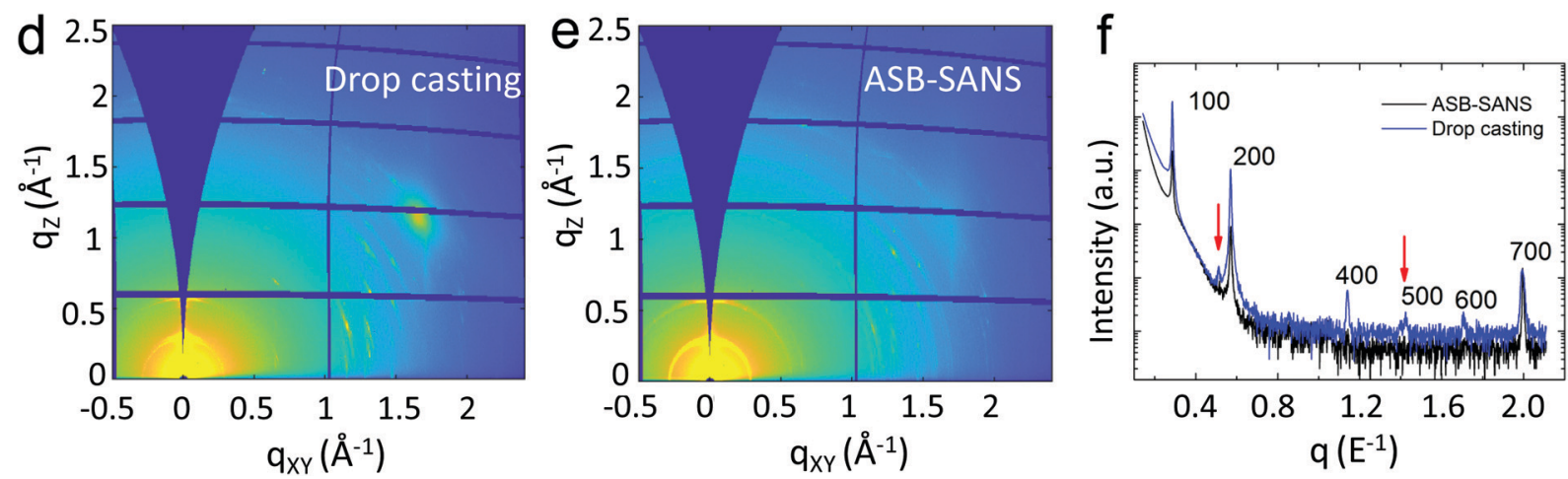

Fig. 3 2D-GIXRD images and specular scans of thin deposits of control samples of 1 prepared by drop casting and ASB-SANS using as solvent chloroform $(a-c)$ and toluene $(d-f)$. 
the nature of solution 1 (see time laps of the last step of the process in Fig. S1 in the ESI $\dagger$ ). ${ }^{27-30}$

Summarizing, we have demonstrated the capability to obtain almost full selectivity in the crystallization of a precise polymorph of a model molecule by using ASB-SANS. The method is fully chemical and does not need any external tools. It exploits at the same time the establishment of quasi-equilibrium conditions and the mesoscopic confinement for the formation of the polymorph. Remarkably, ASB-SANS eliminates the problem of separating the polymorph from the confining matrix, as the medium used for the confinement sublimates away at the end of the process. ASB-SANS is a single step, simple, versatile and reproducible process, and it allows one to grow controlled molecular nanostructures of a selected polymorph. Since the process here described does not rely on specific interactions between the polymorph-capable molecules and the matrix, the method is of general application, and we expect it to be applied to a large variety of polymorphic materials. At this stage, we did not consider the scalability of the process. We believe that for an upscaling of the process, a system able to recycle the sublimating compound must be used.

We thank Lucia Maini for useful discussion. We are grateful for the financial support provided by the Italian Minister of instruction, university and research. National project PRIN "Next generation of molecular and supramolecular machines: towards functional nanostructured devices, interfaces, surfaces and materials (NEMO)" Prot. 20173L7W8K.

\section{Conflicts of interest}

There are no conflicts to declare.

\section{Notes and references}

$1 \mathrm{~J}$. Bernstein, Polymorphism in molecular crystals, Oxford University Press, New York, 2002.

2 G. R. Desiraju, Cryst. Growth Des., 2008, 8, 3-5.

3 D. Gentili, M. Gazzano, M. Melucci, D. Jones and M. Cavallini, Chem. Soc. Rev., 2019, 48, 2502-2517.

4 S. Galindo, A. Tamayo, F. Leonardi and M. Mas-Torrent, Adv. Funct. Mater., 2017, 27, 1700526.

5 C. M. Fernández-Posada, A. Castro, J.-M. Kiat, F. Porcher, O. Peña, M. Algueró and H. Amorín, Nat. Commun., 2016, 7, 12772.

6 A. Llinàs and J. M. Goodman, Drug Discovery Today, 2008, 13, 198-210.

7 L. Maini, F. Gallino, M. Zambianchi, M. Durso, M. Gazzano, K. Rubini, D. Gentili, I. Manet, M. Muccini, S. Toffanin, M. Cavallini and M. Melucci, Chem. Commun., 2015, 51, 2033-2035.
8 A. O. F. Jones, B. Chattopadhyay, Y. H. Geerts and R. Resel, Adv. Funct. Mater., 2016, 26, 2233-2255.

9 D. Gentili, M. Durso, C. Bettini, I. Manet, M. Gazzano, R. Capelli, M. Muccini, M. Melucci and M. Cavallini, Sci. Rep., 2013, 3, 2581.

10 J. Chen, M. Shao, K. Xiao, A. J. Rondinone, Y.-L. Loo, P. R. C. Kent, B. G. Sumpter, D. Li, J. K. Keum, P. J. Diemer, J. E. Anthony, O. D. Jurchescu and J. Huang, Nanoscale, 2014, 6, 449-456.

11 A. O. F. Jones, Y. H. Geerts, J. Karpinska, A. R. Kennedy, R. Resel, C. Röthel, C. Ruzié, O. Werzer and M. Sferrazza, ACS Appl. Mater. Interfaces, 2015, 7, 1868-1873.

12 J.-P. Brog, C.-L. Chanez, A. Crochet and K. M. Fromm, RSC Adv., 2013, 3, 16905-16931.

13 A. M. Hiszpanski, R. M. Baur, B. Kim, N. J. Tremblay, C. Nuckolls, A. R. Woll and Y.-L. Loo, J. Am. Chem. Soc., 2014, 136, 15749-15756.

14 G. Giri, R. Li, D.-M. Smilgies, E. Q. Li, Y. Diao, K. M. Lenn, M. Chiu, D. W. Lin, R. Allen, J. Reinspach, S. C. B. Mannsfeld, S. T. Thoroddsen, P. Clancy, Z. Bao and A. Amassian, Nat. Commun., 2014, 5, 3573.

15 H. Chung and Y. Diao, J. Mater. Chem. C, 2016, 4, 3915-3933.

16 D. Gentili, F. Valle, C. Albonetti, F. Liscio and M. Cavallini, Acc. Chem. Res., 2014, 47, 2692-2699.

17 A. V. Trask and W. Jones, in Organic Solid State Reactions, ed. F. Toda, 2005, vol. 254, pp. 41-70.

18 J.-M. Ha, J. H. Wolf, M. A. Hillmyer and M. D. Ward, J. Am. Chem. Soc., 2004, 126, 3382-3383.

19 Q. Jiang and M. D. Ward, Chem. Soc. Rev., 2014, 43, 2066-2079.

20 A. Tamayo, S. Riera-Galindo, A. O. F. Jones, R. Resel and M. MasTorrent, Adv. Mater. Interfaces, 2019, 6, 1900950.

21 B. D. Hamilton, J.-M. Ha, M. A. Hillmyer and M. D. Ward, Acc. Chem. Res., 2012, 45, 414-423.

22 P. Maheshwari, D. Dutta, S. K. Sharma, K. Sudarshan, P. K. Pujari, M. Majumder, B. Pahari, B. Bandyopadhyay, K. Ghoshray and A. Ghoshray, J. Phys. Chem. C, 2010, 114, 4966-4972.

23 Y. Diao, K. M. Lenn, W.-Y. Lee, M. A. Blood-Forsythe, J. Xu, Y. Mao, Y. Kim, J. A. Reinspach, S. Park, A. Aspuru-Guzik, G. Xue, P. Clancy, Z. Bao and S. C. B. Mannsfeld, J. Am. Chem. Soc., 2014, 136, 17046-17057.

24 M. Cavallini, Z. Hemmatian, A. Riminucci, M. Prezioso, V. Morandi and M. Murgia, Adv. Mater., 2012, 24, 1197-1201.

25 M. Melucci, M. Zambianchi, L. Favaretto, V. Palermo, E. Treossi, M. Montalti, S. Bonacchi and M. Cavallini, Chem. Commun., 2011, 47, 1689-1691.

26 M. Cavallini, A. Calo, P. Stoliar, J. C. Kengne, S. Martins, F. C. Matacotta, F. Quist, G. Gbabode, N. Dumont, Y. H. Geerts and F. Biscarini, Adv. Mater., 2009, 21, 4688-4691.

27 A. Fraleoni-Morgera, Small, 2011, 7, 321-325.

28 A. Fraleoni-Morgera, G. Palma and J. R. Plaisier, RSC Adv., 2013, 3, 15664-15669.

29 J. Mohanraj, L. Puzzi, E. Capria, S. Corvaglia, L. Casalis, L. Mestroni, O. Sbaizero and A. Fraleoni-Morgera, Mater. Sci. Eng., C, 2016, 62, 301-306.

30 I. H. Eryilmaz, J. Mohanraj, S. Dal Zilio and A. Fraleoni-Morgera, Sci. Rep., 2017, 7, 10526.

31 M. Durso, C. Bettini, A. Zanelli, M. G. Lobello, F. De Angelis, V. Biondo, D. Gentili, R. Capelli, M. Cavallini, M. Muccini and M. Melucci, Org. Electron., 2013, 14, 3089-3097.

32 M. Cavallini and M. Melucci, ACS Appl. Mater. Interfaces, 2015, 7, 16897-16906.

33 C. Cappuccino, L. Catalano, F. Marin, G. Dushaq, G. Raj, M. Rasras, R. Rezgui, M. Zambianchi, M. Melucci, P. Naumov and L. Maini, Cryst. Growth Des., 2020, DOI: 10.1021/acs.cgd.9b01281. 